\title{
A Deep Learning and GIS Approach for the Optimal Positioning of Wave Energy Converters
}

\author{
Georgios Batsis ${ }^{1}$, Panagiotis Partsinevelos ${ }^{2, *(D)}$ and Georgios Stavrakakis ${ }^{1}$ \\ 1 School of Electrical and Computer Engineering, Technical University of Crete, 73100 Chania, Greece; \\ georgebatsis95@gmail.com (G.B.); gstavr@electronics.tuc.gr (G.S.) \\ 2 Senselab Research, School of Mineral Resources Engineering, Technical University of Crete, \\ 73100 Chania, Greece \\ * Correspondence: ppartsi@mred.tuc.gr
}

check for updates

Citation: Batsis, G.; Partsinevelos, P.; Stavrakakis, G. A Deep Learning and GIS Approach for the Optimal Positioning of Wave Energy Converters. Energies 2021, 14, 6773. https://doi.org/10.3390/en14206773

Academic Editor:

Mohamed Benbouzid

Received: 11 August 2021

Accepted: 10 October 2021

Published: 17 October 2021

Publisher's Note: MDPI stays neutral with regard to jurisdictional claims in published maps and institutional affiliations.

Copyright: (c) 2021 by the authors. Licensee MDPI, Basel, Switzerland. This article is an open access article distributed under the terms and conditions of the Creative Commons Attribution (CC BY) license (https:// creativecommons.org/licenses/by/ $4.0 /)$.
Abstract: Renewable Energy Sources provide a viable solution to the problem of ever-increasing climate change. For this reason, several countries focus on electricity production using alternative sources. In this paper, the optimal positioning of the installation of wave energy converters is examined taking into account geospatial and technical limitations. Geospatial constraints depend on Land Use classes and seagrass of the coastal areas, while technical limitations include meteorological conditions and the morphology of the seabed. Suitable installation areas are selected after the exclusion of points that do not meet the aforementioned restrictions. We implemented a Deep Neural Network that operates based on heterogeneous data fusion, in this case satellite images and time series of meteorological data. This fact implies the definition of a two-branches architecture. The branch that is trained with image data provides for the localization of dynamic geospatial classes in the potential installation area, whereas the second one is responsible for the classification of the region according to the potential wave energy using wave height and period time series. In making the final decision on the suitability of the potential area, a large number of static land use data play an important role. These data are combined with neural network predictions for the optimizing positioning of the Wave Energy Converters. For the sake of completeness and flexibility, a Multi-Task Neural Network is developed. This model, in addition to predicting the suitability of an area depending on seagrass patterns and wave energy, also predicts land use classes through Multi-Label classification process. The proposed methodology is applied in the marine area of the city of Sines, Portugal. The first neural network achieves 98.7\% Binary Classification accuracy, while the Multi-Task Neural Network $97.5 \%$ in the same metric and $93.5 \%$ in the F1 score of the Multi-Label classification output.

Keywords: wave energy converters; deep neural networks; renewable energy sources; spatial planning; sentinel satellite imagery

\section{Introduction}

Currently, the multifaceted phenomenon of climate change is a matter of increasing concern. Despite the fact that the rapid technological progress leads to the improvement of human well-being, some of the industrial sectors are responsible for a significant part of greenhouse gas emissions. More specifically, industry emits $37 \%$ of the total gas emissions and a significant part of this percentage represents the environmental cost of producing the required electrical energy for the operation of industrial activities [1]. The need to meet the growing energy demand combined with the least possible environmental consequences, leads to the emergence of renewable energy plants [2]. A challenge for Europe is the ability to generate electricity from renewable sources at high and increasing, over time, levels. Particularly, the objective of EU is to cover the $32 \%$ of the total European energy demand from renewable sources by 2030 and to reduce the greenhouse gas emissions by $40 \%$ compared to 1999 [3]. It is necessary for various renewable energy plants to be 
developed in order to achieve the aforementioned objectives. Many countries are looking for an efficient way to use the ocean as a renewable energy source. Wave energy systems are characterized by high efficiency and their contribution to Europe's energy demand is estimated to be $15 \%$ by 2050 [4]. Towards this end, Wave Energy Converters (WEC) are systems that exploit the wave energy, converting it into electricity and they can be installed either independently or in combination with Offshore Wind Generators [5].

Optimal positioning of WECs, is a crucial issue for the marine environment, human activities and the wave energy potential. Suitable areas for WEC installation are selected after the exclusion of points that do not meet certain restrictions. WEC planning limitations are determined by a specific set of rules included in the Marine Spatial Planning (MSP) [6]. In this way, the negative aspect of these systems on the marine activities and the natural environment (i.e., algae, beaches, protected areas and coastal agriculture activities) is prevented. In addition, it is necessary to exclude regions in which the operation of WEC is not efficient (i.e., weather conditions and seabed morphology). Thus, WEC positioning constitutes a decision-making problem in which multiple constraints should be taken into account.

The first step for the optimal positioning of WEC devices is the assessment of the wave energy resource. The most recent research in potential wave energy characterization is based on in situ, satellite and reanalysis data or wave model simulations. Farkas et al. [7], used the third-generation wave model (WAM) combined with altimetry data and characterised the annual, monthly and seasonal wave energy for two specific devices. Authors compared Wave Energy Potential at seven different locations. Additionally, Nilsson et al. [8] utilized WAM evaluated by bouy data and calculated the wave energy potential of the Exclusive Economic Zone (EEZ). They performed wave energy classification in five categories comparing the energy resource of selected locations to the mean wave energy and standard deviation of other sites at similar distance to the shoreline. An additional wave model is the Simulating Waves Nearshore (SWAN) which is developed at Delft University of Technology. Veigas et al. [9] utilized SWAN to find out the offshore wave conditions near the shoreline. Wave energy resource characterization is carried out according to the wave power matrix of the Sea Slot-cone Generator (SSG) data [10] of an offshore bouy and the calculation of the mean annual energy values. Amarouche et al. [11] developed a historical dataset using the SWAN model. Authors classified the potential wave energy flux through the calculation of different temporal variations of wave energy, the probability of distribution, the wave energy development index and the yearly wave energy. Fairley et al. [12] presented a novel method for the wave energy resource characterization. One of the main results of this study are the consequences of the different temporal variability at wave power time series of two locations. Despite the fact that these time series have the same mean value, the standard deviation is different. Thus, the traditional methods for the wave energy resource assessment (i.e., Annual Wave Energy Flux) lead to inefficient results because they cannot handle temporal variability differences. The authors proposed a novel clustering-based method to evaluate wave height and period time series.

Additional to the aforementioned analysis, optimal locations for WEC installation are carried out through geospatial analysis. Researchers and practitioners use Geographic Information Systems (GIS), Multiple-criteria, or a combination of them to deal with optimal positioning of Renewable Energy Systems. Aydin et al. [13], used a GIS-based method in order to find optimal locations for hybrid renewable energy plants in Turkey. Regarding the WEC positioning, Castro-Santos et al. [14], proposed a GIS-based approach under which data were collected for the corresponding geospatial limitations, the seabed morphology, as well as the meteorological conditions and developed four GIS tools. After the combination of corresponding GIS layers, the final decision is made by identifying the available areas on the resulting map. Galparsoro Iza et al. [15], developed a decision making system that combines the MSP approach and GIS. Authors calculated Suitability Index in order to find optimal locations for WEC installation. Apart from the aforementioned methods, some researchers have used Multiple-criteria decision-making (MCDM) systems. Vasileiou 
et al. [16] developed a combined GIS-MCDM system to select cites for a hybrid offshore wind-wave farm in Greece. Ghosh et al. [17] proposed a system for WEC positioning. Limitations of WEC planning are defined with the corresponding weights and the MCDM System derives the Feasibility Index (FI) for each of the potential installation regions. An Artificial Neural Network (ANN) is also used to predict the FI value according to the criteria.

The state-of-the-art methods for wave energy resource assessment and for optimal positioning of WEC including geospatial analysis are summarized on Tables 1 and 2, respectively. As a rule, both geospatial and technical limitations criteria for WEC positioning can be modeled using the traditional GIS-based methods. However, in some cases it is essential to examine restrictions related to dynamically changing patterns in marine areas. This is the major limitation of the state of the art methods for WEC positioning, including geospatial analysis (Table 2). For instance, algae are important for the environmental balance and cannot be identified using a GIS database. Geographic datasets entail only historical algae presence records for specific dates. Towards this end, Effrosynidis et al. [18] proposed a Machine Learning (ML) based method to identify seagrass presence according to environmental variables. In addition, satellite imagery is an efficient method for seagrass mapping [19] and Deep Neural Networks (DNN) are used to automate this process. More precisely, Li et al. [20] trained a Convolutional Neural Network (CNN) for the seagrass segmentation of satellite images. Thus, in our study we use Deep Learning methods and remote sensing data in order to identify dynamically changing patterns such as algae in marine areas. Last but not least, relying on Fairley's et al. [12] study, one may highlight the major limitation of the state of the art methods for wave energy resource characterization. According to their results, traditional studies lead to inefficient results due to the difference in time variability of separated locations. In order to deal with this limitation, we expand Fairley's et al. [12] method for the assessment of wave energy potential through the generation of a wave energy dataset with the corresponding suitability labels of wave height and period time series, in order to implement a Deep Learning based algorithm for time series classification. Using this method, we handle differences of temporal variability and we are able to use the proposed model for other case studies.

Based on the above information, in the present study we address the optimal positioning of WEC establishments using a DNN approach. Machine learning techniques are widely used in Renewable Energy Systems in order to estimate the maximum energy production [21], to predict production and load time series [22-24] or to identify potential space for new installations such as photovoltaic systems $[25,26]$. The heterogeneity and dynamic nature of data and the desire for automation are the main driving forces to undertake the proposed methodology. Thus, we divide the necessary data into static (i.e., land use classes) and dynamic (i.e., algae and wave energy potential). We propose a Deep Learning-based decision system that detects dynamic geospatial limitations, while it evaluates the wave energy potential with respect to time variability. Particularly, we developed a DNN that operates on heterogeneous data fusion [27], in this case satellite images and time series of meteorological data. This fact implies the definition of a two-branches architecture. The branch that is trained with image data provides the localization of dynamic geospatial classes in the potential installation area, whereas the second one is responsible for the classification of the region according to the potential wave energy. The proposed Neural Network is the core of the system that we implemented to automate the process of WEC optimal positioning. Our system operates in two modes: in Mode I, the image recognition branch of DNN only detects algae. The land use classes are received from a land use database and are combined with DNN predictions for the WEC optimizing positioning. 
Table 1. Related work for the assessment of wave energy resource.

\begin{tabular}{|c|c|c|c|}
\hline Authors & Method & $\begin{array}{l}\text { Wave Energy Resource } \\
\text { Assessment }\end{array}$ & Results \\
\hline Farkas et al. & $\begin{array}{l}\text { Numerical Method and } \\
\text { Wave Model }\end{array}$ & $\begin{array}{l}\text { Annual, Monthly and Seasonal } \\
\text { Wave Energy. Comparison with } \\
\text { Offshore Wind Energy potential. }\end{array}$ & $\begin{array}{l}\text { Authors compared Wave Energy } \\
\text { Potential at seven different locations. } \\
\text { Highest values are obtained during } \\
\text { winter and lowest at summer. }\end{array}$ \\
\hline Nilsson et al. & $\begin{array}{l}\text { Numerical Method and } \\
\text { Wave Model }\end{array}$ & $\begin{array}{l}\text { Wave energy resource } \\
\text { classification through the } \\
\text { comparison of mean wave energy } \\
\text { potential and its standard } \\
\text { deviation of sites at similar } \\
\text { distance to the shoreline. }\end{array}$ & $\begin{array}{l}\text { Areas with the highest Wave Energy } \\
\text { potential located within the Exclusive } \\
\text { Economic Zone of Sweden. }\end{array}$ \\
\hline Veigas et al. & $\begin{array}{l}\text { Numerical Method, Wave } \\
\text { Model and SSG Wave } \\
\text { Power Matrix }\end{array}$ & $\begin{array}{l}\text { Comparison with offshore buoy } \\
\text { data and SSD Wave Power Matrix. }\end{array}$ & $\begin{array}{l}\text { Authors selected the three best } \\
\text { locations. In addition, they calculated } \\
\text { the WEC capacity factor ( } 33 \% \text { or } \\
2628 \text { equivalent hours). }\end{array}$ \\
\hline Amarouche et al. & $\begin{array}{l}\text { Numerical Method and } \\
\text { Wave Model }\end{array}$ & $\begin{array}{l}\text { Temporal variations of different } \\
\text { scales, probability of distribution, } \\
\text { wave energy development index } \\
\text { and annual wave energy. }\end{array}$ & $\begin{array}{l}\text { Authors characterized Eastern } \\
\text { Algerian coast as one of the highest } \\
\text { energy potential locations in } \\
\text { the Mediterranean. }\end{array}$ \\
\hline Fairley et al. & Multivariate Clustering & $\begin{array}{l}\text { K-means for wave energy } \\
\text { resource clustering. }\end{array}$ & $\begin{array}{c}\text { Traditional methods for the wave } \\
\text { energy resource assessment (i.e., } \\
\text { Annual Wave Energy Flux) lead to } \\
\text { inefficient results because they cannot } \\
\text { handle temporal variability. }\end{array}$ \\
\hline
\end{tabular}

Table 2. Related work for WEC positioning assuming geospatial analysis.

\begin{tabular}{|c|c|c|c|c|}
\hline Authors & Plant & Method & Geospatial Analysis & $\begin{array}{l}\text { Renewable Energy } \\
\text { Resource Assessment }\end{array}$ \\
\hline Aydin et al. & $\begin{array}{l}\text { Hybrid Solar and } \\
\text { Wind Farms }\end{array}$ & GIS and MCDM & $\begin{array}{c}\text { Data Collection, } \\
\text { Objectives as fuzzy sets, } \\
\text { Environmental } \\
\text { performance index }\end{array}$ & $\begin{array}{c}\text { Energy } \\
\text { Performance Index }\end{array}$ \\
\hline Castro-Santos et al. & $\begin{array}{l}\text { Hybrid Offshore Wind } \\
\text { and Wave Farms }\end{array}$ & GIS & $\begin{array}{l}\text { Data Collection and } \\
\text { GIS techniques } \\
\text { Data Collection }\end{array}$ & $\begin{array}{l}\text { Annual Energy } \\
\text { Production }\end{array}$ \\
\hline Vasileiou et al. & $\begin{array}{l}\text { Hybrid Offshore Wind } \\
\text { and Wave Farms }\end{array}$ & GIS and MCDM & $\begin{array}{c}\text { Exclusion of unsuitable } \\
\text { areas, AHP for site } \\
\text { selection }\end{array}$ & Average Power \\
\hline Ghosh et al. & Wave Farm & $\begin{array}{l}\text { MCDM and ANN that } \\
\text { predicts index for } \\
\text { decision-making. }\end{array}$ & Historical Data & $\begin{array}{l}\text { Significant Wave } \\
\text { Height and Wind } \\
\text { Speed Average }\end{array}$ \\
\hline Galparsoro Iza et al. & Wave Farm & $\begin{array}{c}\text { Marine Spatial } \\
\text { Planning and GIS. } \\
\text { Suitability Index. }\end{array}$ & $\begin{array}{l}\text { Data Collection and } \\
\text { GIS techniques }\end{array}$ & Local Wave Atlas \\
\hline
\end{tabular}

On the other hand, in Mode II, the image recognition branch classifies land use as well as the algae patterns. Thus, the potential regions classified as suitable or as not suitable for WEC installation are exclusively processed via DNN predictions. In both cases, final classification is based upon feature extraction from both image and time series datasets. Overall, the main contributions of this paper include:

- $\quad$ Automation of WEC optimal positioning through Deep Learning algorithms.

- Recognition of dynamically changing patterns-geospatial WEC planning constraints.

- Wave energy potential assessment using time series classification.

- Recognition of dynamic spatial constrains and characterization of wave height and period time series simultaneously via Data Fusion DNN. 
In the next section, the methodology and architecture of both implementation modes are described, along with the data used. Section 3 presents the results of both methodological modes and concludes with a real-life case study paradigm. Section 4 discusses the findings of the proposed work and suggests further development paths.

\section{Methodology}

In this section, we present the proposed methodology for WEC positioning. At first, we describe how we developed our heterogeneous dataset. More precisely, we created a Geographic Information Tool with the usage of which we receive satellite imagery and time series data. In addition, we present the labeling method, in order to develop the training, validation and test dataset for Deep Learning models. The Deep Learning algorithm consists of two modes and for each of them two different DNN architectures are shown. In conclusion, we can use this algorithm after the training process to develop a decision-making system for WEC positioning. All procedure was carried out with the use of our code written in Python programming language and GeoPandas, GDAL and Tensorflow libraries.

\subsection{Dataset Generation-Geographic Information Tool}

The extraction of specific geographic datasets is necessary in order to implement the proposed method. We developed a Geographic Information Tool, which is necessary to generate the training dataset and apply the methodology to the selected area of interest. In its general form, this tool uses bounding box coordinates of the area of interest as an input and defines the grid of the potential WEC installation points, as well as it incorporates the necessary data. The flowchart of this tool is shown in Figure 1. Initially, we acquire the Sentinel-2 Tile [28] for the corresponding geographical coordinate using the Open Access Hub API. Then, we define the grid of the potential points for WEC installation and we create the georeferenced patch of Sentinel-2 images for each of the patches using the geometric buffer operation. We receive bathymetric data from GEBCO [29] and 12-year Wave Height and Period time series using $3 \mathrm{~h}$ time-step from ERA-5 dataset [30] via the Climate Data Store (CDS) API. In addition, we interpolate bathymetric and time series data to our grid, via the CDS Toolbox. For nearshore potential regions we extract land use classes and their polygons from Corine Land Cover (CLC) dataset [31].

The output of our Geographic Information Tool is two databases connected via an information file. The first database contains the georeferenced image patches and the other one the historical wave height and period time series. In Figure 2, an example of a potential WEC installation point is shown. In time series plot the $x$-axis represents the 3 -h time step for the past 12 years and $y$-axis represents the values of Significant Wave Height (meters) and Peak Wave Period (seconds). Despite the fact that image patches and time series are stored to the corresponding database, the water depth and land use classes are stored in the information file.

Generation of training dataset involves the labeling process of both satellite images and time series. More precisely, we must assign labels to image patches according to algae presence or absence. For this purpose, we use the algae presence observations from UNEP-WCMC dataset [32,33]. By extracting the potential WEC installation points with the aforementioned tool, the event dates from UNEP-WCMC records are used for the region of South Italy to acquire the corresponding Sentinel images. In this way, we used spatial intersection operation between seagrass and patches polygons to assign labels to potential WEC installation regions. On the other hand, time series labeling process is crucial for the assessment of the wave energy potential. In this paper, the novel method of Fairley et al. [12] is used to assign labels about the wave energy. Particularly we implemented the W-based approach. Considering the Significant Wave Height $\left(H_{S}\right)$ and Peak Wave Period $\left(T_{p}\right)$ we calculate the Coefficient of Variation $(C V)$ of $\overline{H_{s}^{2}}, \overline{T_{p}}$. Precise, $C V$ is the ratio 
of standard deviation of a variable and its mean value. For each of the time series the following variables are calculated:

$$
\begin{gathered}
\overline{H_{s}^{2}}, \overline{T_{p}} \\
C V_{H_{s}^{2}}=\frac{\sigma_{H_{s}^{2}}}{\overline{H_{s}^{2}}} \\
C V_{T_{p}}=\frac{\sigma_{T_{p}}}{\overline{T_{p}}}
\end{gathered}
$$
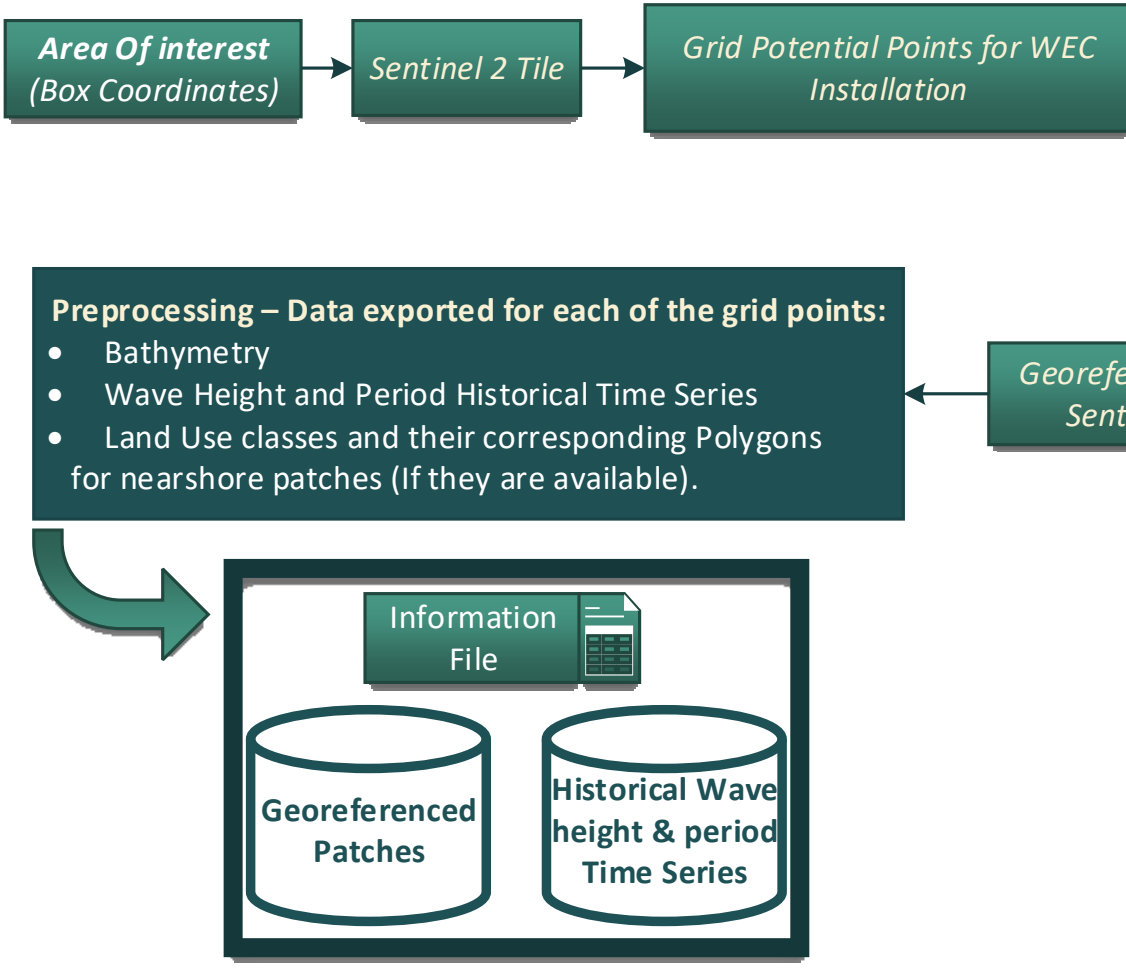

Figure 1. Flowchart of Geographic Information Tool.

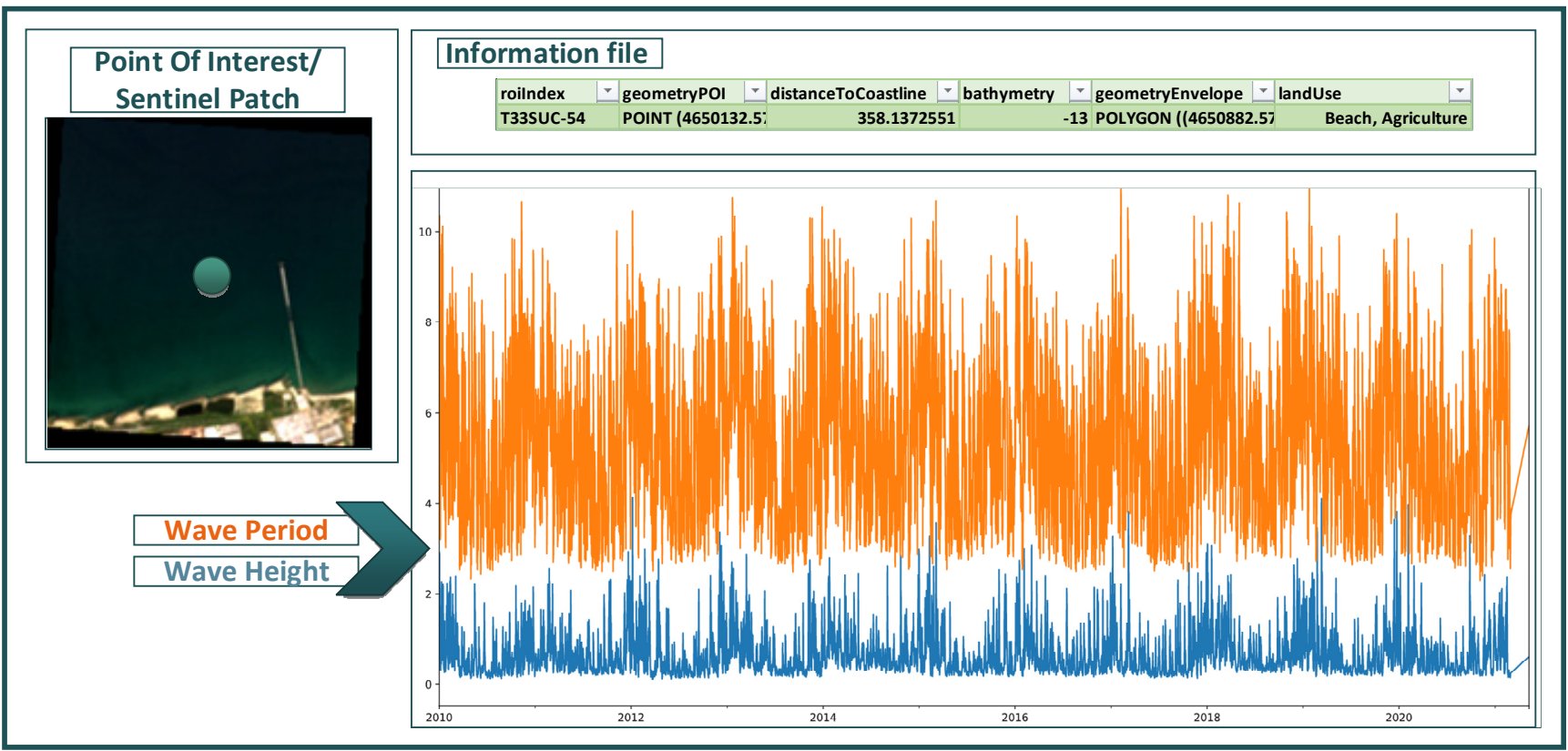

Figure 2. Example of a potential WEC installation point. 
The aforementioned variables are used to cluster time series using K-Means $(\mathrm{K}=6)$. According to Significant Wave Height cluster-mean we can sort clusters from 1 to 6 or low wave energy to high wave energy potential, respectively. Thus, we define the clusters 1 to 3 as unsuitable for WEC installation and the clusters from 4 to 6 as suitable. Finally, we can combine the above information in order to assign labels to potential WEC installation points as follows:

- High Wave energy potential and Algae Absence means Suitable Area;

- High Wave energy potential and Algae Presence means Unsuitable Area;

- Low Wave energy potential and Algae Absence means Unsuitable Area;

- Low Wave energy potential and Algae Presence means Unsuitable Area.

\subsection{Deep Learning Algorithm}

The proposed Deep Learning algorithm is the core of the system that we implemented to automate the process of WEC optimal positioning. As mentioned above, our system operates in two modes: In Mode I, we developed a Data Fusion based Neural Network. The image recognition branch only detects algae. The land use classes are received from a land use dataset. On the other hand, in Mode II, the image recognition branch classifies land use as well as the algae patterns. Thus, the potential regions classified as suitable or as not suitable for WEC installation is performed exclusively via DNN predictions. In this mode, we have implemented a Multitask Data Fusion based Neural Network. In both modes, we used heterogeneous data fusion techniques because the second branch of proposed architecture classifies wave height and period time series.

The Deep Learning model that we implemented is a Convolutional Neural Network (CNN), which is widely used for image recognition and is trained using the Backpropagation algorithm like the traditional ANN [34-37]. Besides this, CNN are efficient for time series classification [38,39]. Consequently, we developed a Convolutional architecture for each of two branches.

\subsubsection{Data Fusion Based Neural Network}

Consequently, we developed a Convolutional architecture for each of two branches. In order to create Multimodal DNN, its two branches are developed as individual Neural Networks. The reason leading to the specific implementation is to find the optimal architecture for each branch. One of the popular neural networks that work efficiently in the process of identifying marine algae in satellite imagery is the U-Net [20]. This architecture is used for the Semantic Segmentation of an image. However, since we aim to classify images on the presence or absence of algae, an architecture inspired by the Encoder of U-Net is created, due to its efficiency in extracting features from images.

According to Figure 3, the image recognition branch of the Neural Network has three convolutional blocks, in each of which two consecutive Convolutional Layers with the Relu activation function followed by Max Pooling are placed. The number of feature Maps defined per block is 32-64-128, respectively with a $3 \times 3$ filter. The Max Pooling process runs in $2 \times 2$ regions. At the final step, a Global Average Pooling (GAP) layer is used instead of the flatten operation. In this way, the average value is extracted from each feature map of the last convolutional node. GAP can replace the Fully Connected Layer, while helping to avoid overfitting because it reduces the number of training parameters. The time series branch of DNN consists of 1-D Convolutional and Pooling Layers. As it is shown in Figure 3, this branch has one Convolution of 32 feature maps with a kernel size 5, followed by Max Pooling and then another Convolutional Layer with Filter size 7 from which 64 Feature Maps are extracted, followed again by Max Pooling of size 2 . GAP Layer is used instead of Dense Layer too. The extracted features of the two branches are merged via the Concatenation Layer and this layer is fed to a Fully Connected Layer of 256 neurons which are activated via ReLU. Because the task is a Binary Classification problem, the Activation Function that is defined for the final neuron is the Sigmoid. The DNN is implemented on the Tensorflow and Keras Python libraries. 


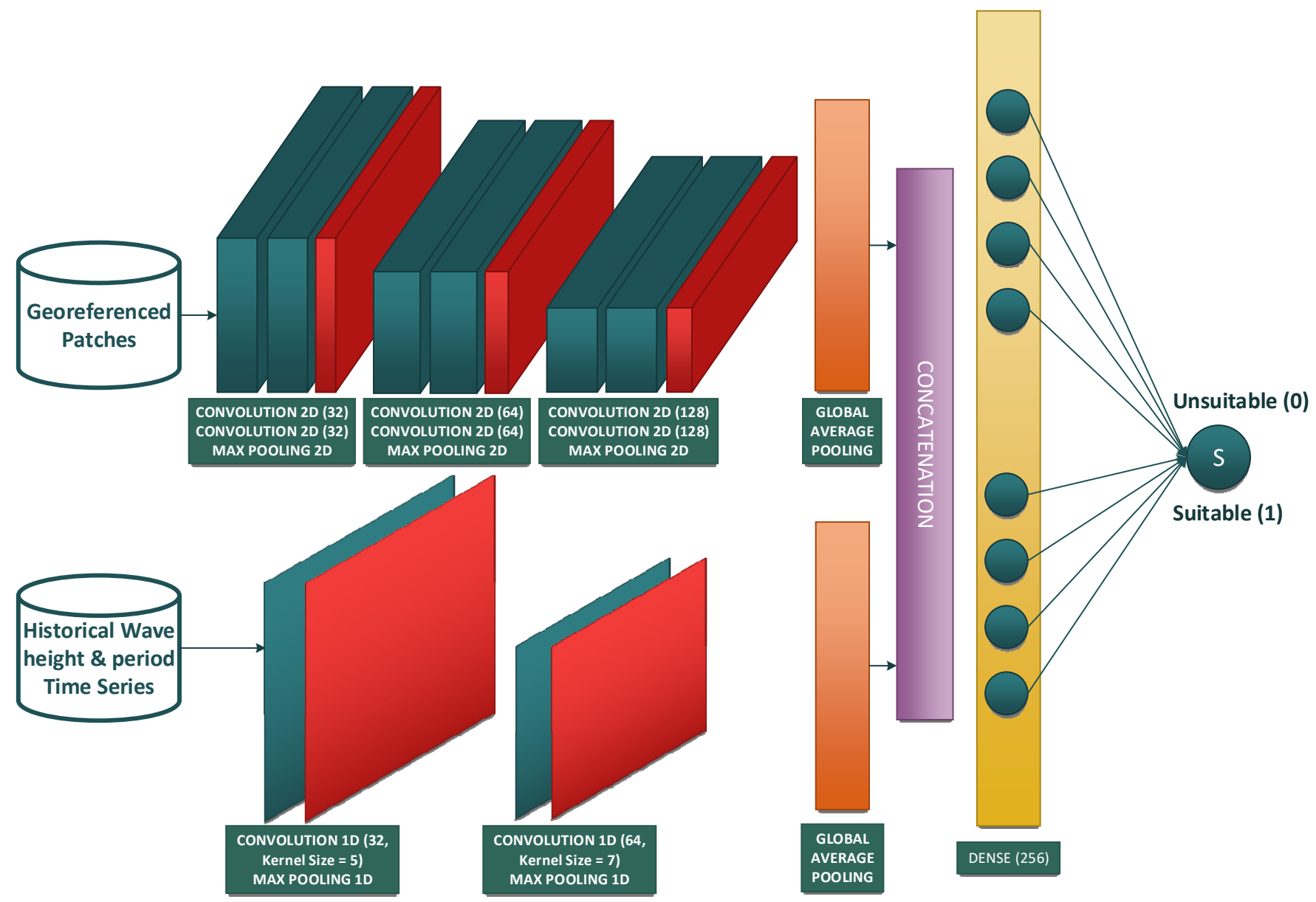

Figure 3. Data fusion Neural Network Architecture-Mode I.

During the training process we need a heterogeneous-data fetching tool, which is developed using the Custom Data Generator of Keras and its input is the information file that connects the two databases (Section 2.1). Satellite images are normalized, dividing each pixel value with 255. The secondary input of Neural Network is a multivariate time series, in particular with two variables, for wave height and period, respectively. Thus, time series are modeled as a 2-D matrix, which consists of two columns, while the number of rows corresponds to the time-steps. Before starting the training process, we split the dataset using $60 \%$ for training, 20\% for validation and $20 \%$ for testing. The validation dataset is fed to the Neural Network at the end of each epoch and when the value of the loss function during the prediction of the data does not improve further, Early Stopping occurs. The Dataset Test is used after the end of the training in order to verify the ability of Neural Network to be generalized. Finally, we use the Adam optimizer [40], 16 batch size, Binary Cross Entropy (BCE) loss function and we estimate model performance according to Accuracy, Precession, Recall and F1 metrics. This Neural Network represents Mode I.

\subsubsection{Multitask Data Fusion Based Neural Network}

In this section, we present the Neural Network that constitutes the core of Mode II. Particularly, the process of land use classification via the image recognition branch is integrated. This implementation requires the adaptation of both the Neural Network architecture and the training dataset. At the first stage, we determined a One-Hot label for each of the potential WEC installation points. The first four cells of the corresponding table describe the geospatial constraints (Agriculture Activities, Beaches/Dunes, Forest Pattern and Algae) and the last cell is the suitability label of wave energy potential. We provide an example of One-Hot label in Figure 4. 

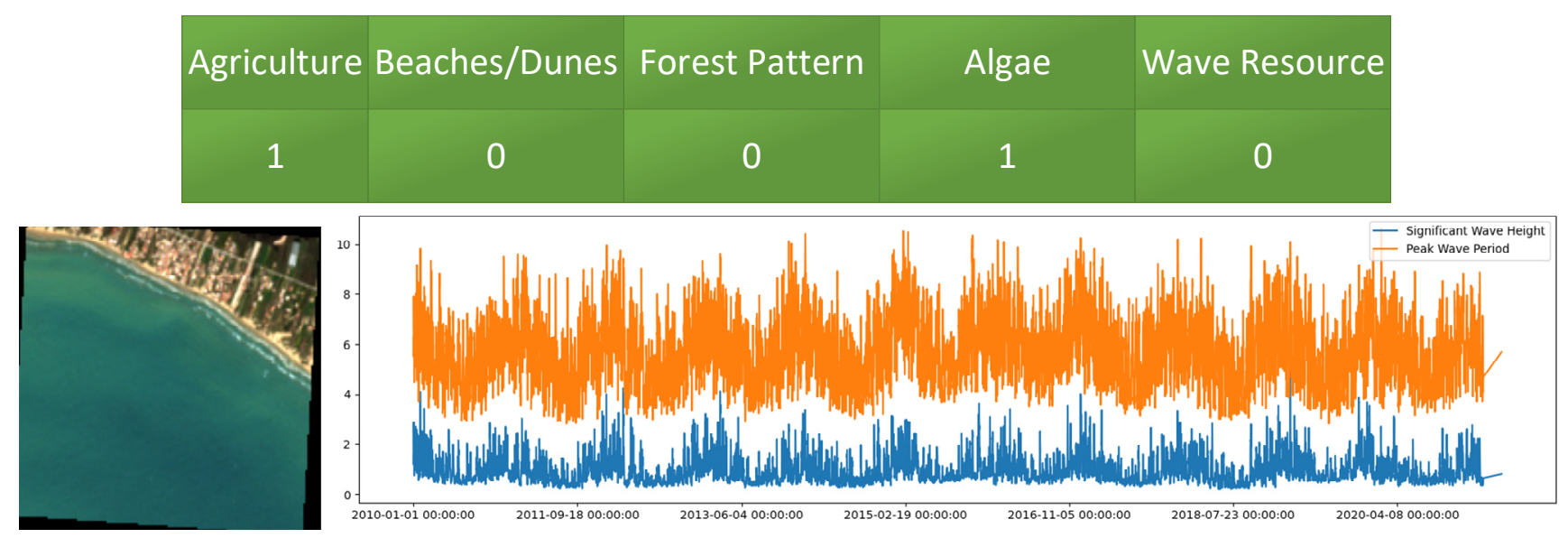

Figure 4. Example of One-Hot vector for unsuitable region.

When the table has the value 1 , then the corresponding class exists in the area. The last cell of the table concerns the wave energy assessment, and values 0 or 1 correspond to low or high wave energy, respectively. Based on the One-Hot table, each point is labelled as suitable or not for WEC installation. In this case, the encoding that implies a suitable point (Label equal to 1 ) is $[0,0,0,0,1]$. In any other case, the potential region is not suitable and the final label is equal to 0 . In Figure 4, an example of an unsuitable region for WEC installation is shown. In this case, we need to build a Neural Network for the solution of two tasks: Multi-label classification and Binary Classification.

When a neural network consists of two outputs, during backpropagation, the common weights are modified in order to optimize two loss functions in parallel. In other words, the model learns to recognize the suitability of a region based on which classes are recognized.

The architecture of Multimodal DNN is modified, initially, as the to the depth of the satellite image recognition branch. In particular, one more Convolution Block is added, and as a result, the number of Feature Maps defined per block are 32-64-128-256, respectively. The time series classification branch remains unchanged. After combining the extracted features from two branches through the Concatenation Layer, a Fully Connected Layer of 256 neurons is used. The first output is for the Binary Classification problem (suitable or not suitable WEC installation point), while the second is used to predict the above One-Hot table. We use BCE loss function on both outputs and we define the Accuracy metric and F1 score in order to estimate the performance of the Binary Classification and Multi-Label classification, respectively. Figure 5 shows the Neural Network Mode II architecture.

\subsection{Optimal Positioning of WECs Using Deep Learning-System Implementation}

As mentioned before, the decision-making system for WEC positioning, is implemented through two modes, which differ in how the information related to the land use of coastal areas is obtained. In Mode I, the satellite imagery branch recognizes exclusively algae patterns which constitutes the dynamic component. Therefore, the prediction of the suitability of each potential point is based on both the presence or absence of algae and the energy availability of the region, which is classified through the time series branch. The output of the Multimodal DNN is combined with the CLC data in order to avoid additional geographic limitations. The variant in Mode II, is the recognition of land use/cover classes by the satellite image branch. In this case, the suitability of each potential WEC installation point is predicted via the DNN.

Common processes for both modes are the definition of potential installation points and the integration of the corresponding data (Section 2.1). In addition, points that are not in the depth range between 10 and $200 \mathrm{~m}$ are excluded. 


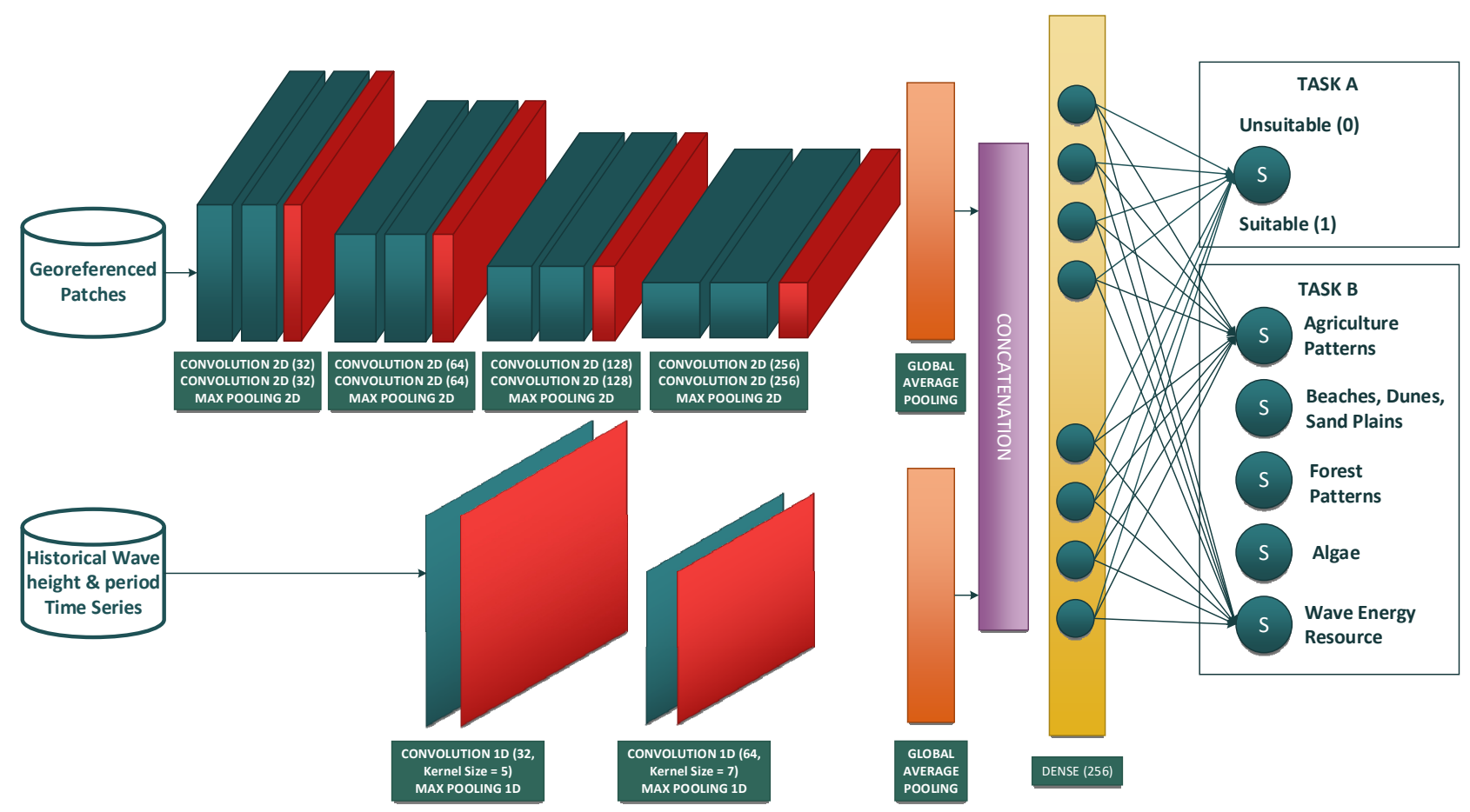

Figure 5. Multitask Data Fusion based Neural Network Architecture.

\subsubsection{Mode I}

The first implementation of the WEC installation positioning system is modeled according to the flowchart in Figure 6. The Data Fusion based DNN of Section 2.2.1 takes the georeferenced Sentinel patch and time series as input and predicts the corresponding binary label. If the latter is zero, then the point is considered as not suitable for WEC installation. In contrast, prediction of suitable points implies both the absence of algae and high wave energy potential. In this case, the system further processes some information in order to make the final decision. In particular, if the potential region is offshore, then it is considered suitable for WEC installation, while if it is a nearshore point, it is required to avoid additional spatial restrictions related to the use/coverage of the closest coastal land. If in the latter beaches, swimming zones, dunes, woodland or agricultural facilities are located, then the point is automatically rejected. Finally, decisions of each of the potential WEC installation sites are combined, in order to construct the suitability map of the overall area of interest.

\subsubsection{Mode II}

The second mode of the WEC optimal positioning methodology contains the Multitask data fusion based Neural Network analyzed in Section 2.2.2 as a core unit. In this way, the decision for each point is taken directly through the corresponding output of DNN, that is, the one that implements the Binary Classification problem. In addition, the output of the Multi-label Classification process is used to monitor how the final decision is formed, since as a result, the land use classes identified, including algae, as well as the energy suitability results are given. The flowchart of this process is in Figure 7. 


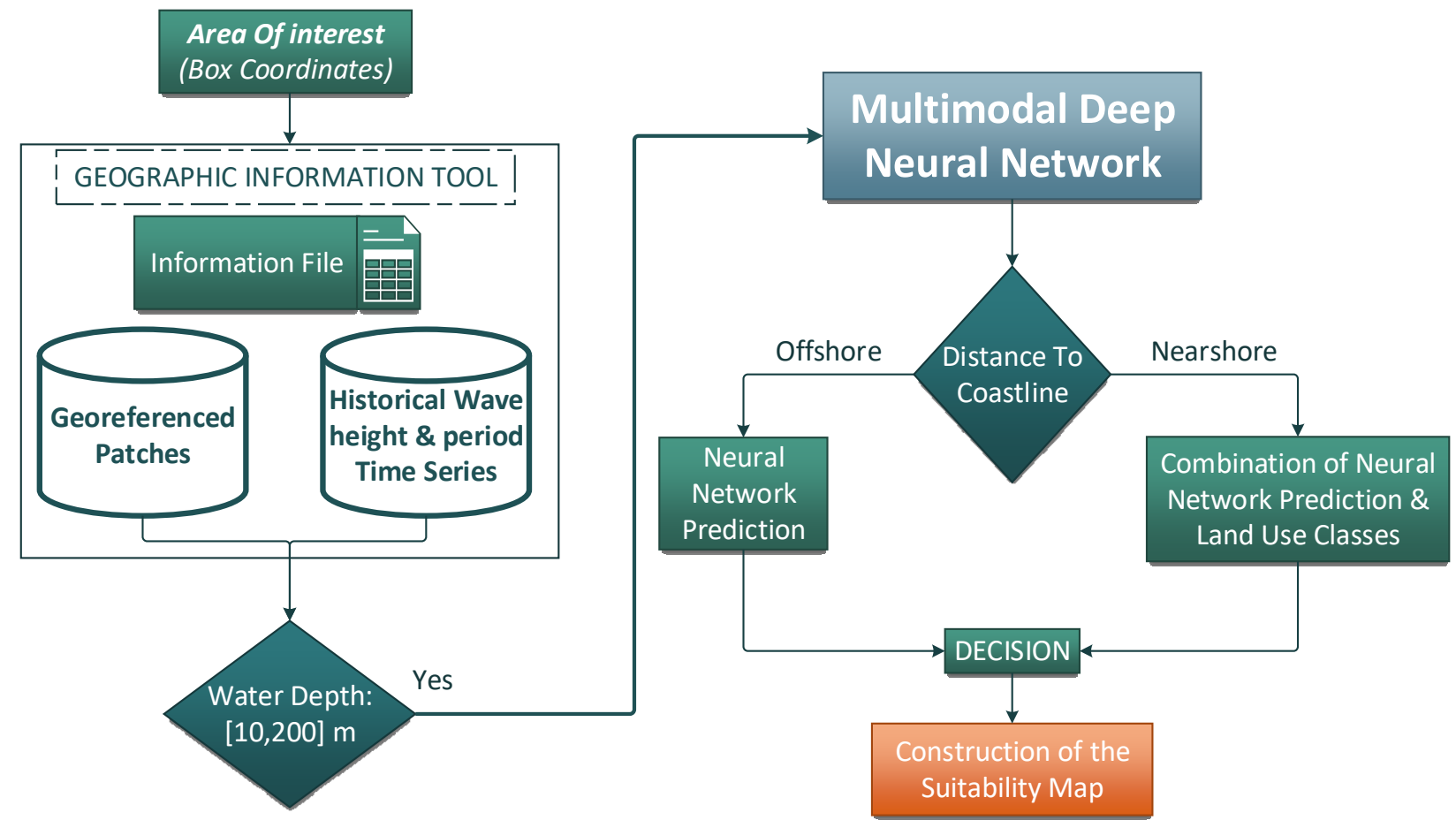

Figure 6. Decision-making system flowchart in Mode I.

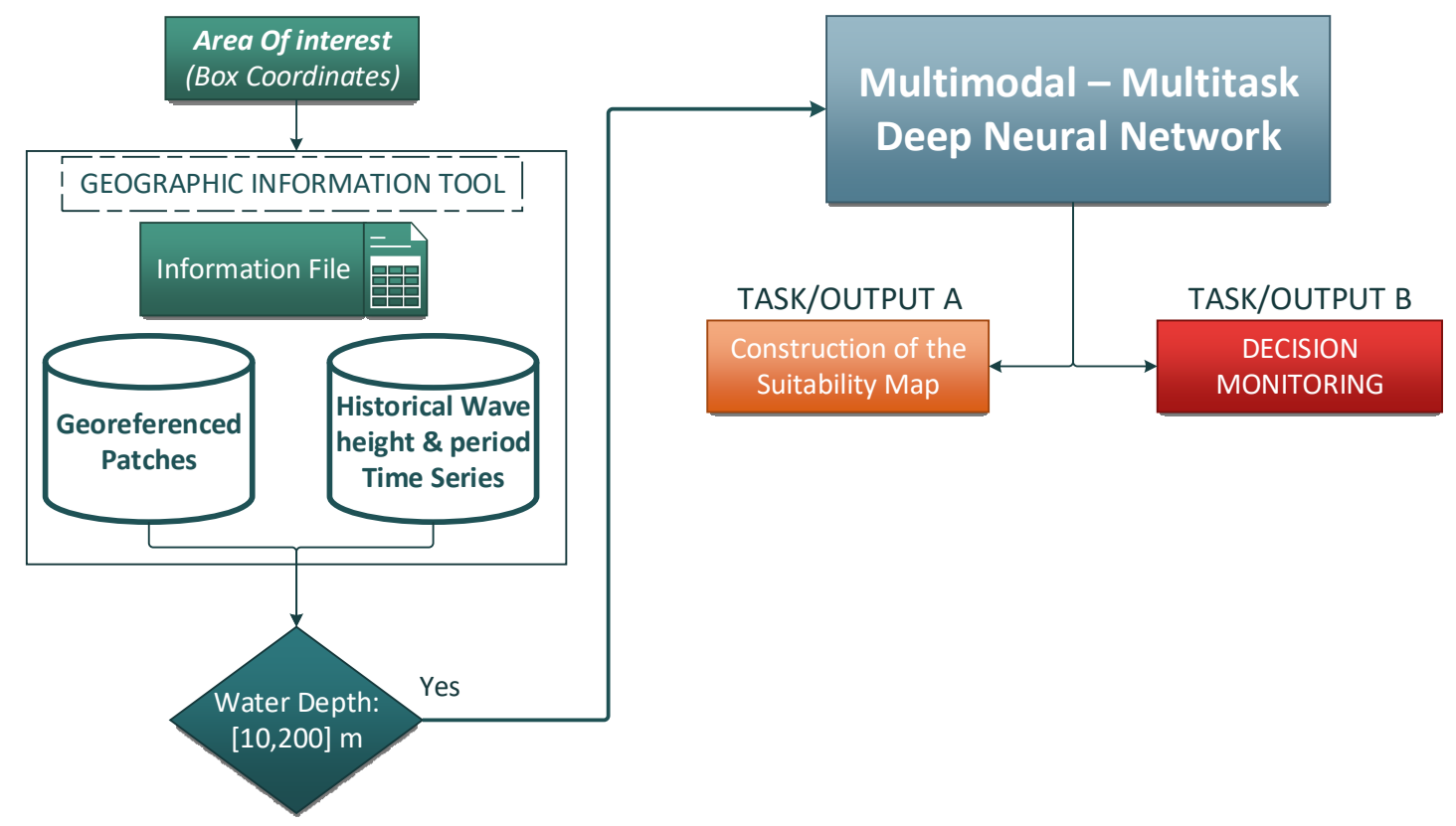

Figure 7. Decision-making system flowchart in Mode II.

\section{Results}

In this section, we present the results of our approach. At first, we analyze the training diagrams and the performance of Deep Learning models. Then, we showcase the proposed methodology to the selected geographical area. We trained our models using the NVDIA GTX 1650 GPU and we used TensorBoard for training process monitoring.

\subsection{Performance Metrics}

In Binary Classification problems, we can estimate the performance of our classifier calculating the number of samples that are classified correctly or incorrectly. If the value of the final neuron output is greater than 0.5 , our sample belongs to positive class, whereas our 
sample belongs to the negative one. Regions that are suitable for the installation of WEC device correspond to class one, whereas the unsuitable locations are marked as samples of negative class. Thus, we calculate True Positive $(T P)$, True Negative $(T N)$, False Positive $(F P)$ and False Negative $(F N)$ classified samples. Using these statistical variables, we can see how many of testing samples are classified correctly or not. In order to visualize the aforementioned statistical approach, we define the Confusion Matrix as follows:

$$
\text { Confusion Matrix }=\left[\begin{array}{cc}
T P & F P \\
F N & T N
\end{array}\right]
$$

Far from these, we examine effectiveness of the proposed model using Recall and Precision. Recall is the ratio of actual positive predictions divided by the number of actual positive samples. In other words, is a metric that shows the effectiveness of our model to accomplish true predictions of positive labels. On the other hand, Precision is the ratio of actual positive samples divided by the number of all positive predictions. Thus, Precision is the proportion of actually correct positive predictions [41].

$$
\begin{gathered}
\text { Recall }=\frac{T P}{T P+F N} \\
\text { Precission }=\frac{T P}{T P+F P}
\end{gathered}
$$

Precision and Recall combined in a single performance metric. F1 score is the harmonic mean of both. We defined $F 1$ as the actual model performance metric during training process. F1 is using in Binary Classification problems [42].

$$
F 1=2 * \frac{\text { Precision } * \text { Recall }}{\text { Precission }+ \text { Recall }}
$$

\subsection{Data Fusion Based Neural Network}

Searching about the optimal architecture of the Data Fusion-Based Neural Network, we implemented each branch of it as an individual DNN. Particularly, we added a sigmoid neuron after the GAP layer. Thus, two binary classifiers have developed, for algae and time series classification, respectively. Compared to other methods for algae classification and detection based on remote sensing data [18,20,43], our approach achieves $98.5 \%$ (Table 3). F1 score on test dataset according to the Confusion Matrix. The equivalent percentage for testing samples of time series classification CNN is $98.8 \%$ (Table 4).

Table 3. Confusion Matrix for algae detection problem.

\begin{tabular}{ccc}
\hline & Predicted-Class 0 & Predicted-Class 1 \\
\hline Actual-Class 0 & TN: 2071 & FP: 14 \\
Actual-Class 1 & FN: 30 & TP: 1959 \\
\hline
\end{tabular}

Table 4. Confusion Matrix for time series classification problem.

\begin{tabular}{ccc}
\hline & Predicted-Class 0 & Predicted-Class 1 \\
\hline Actual-Class 0 & TN: 1053 & FP: 6 \\
Actual-Class 1 & FN: 18 & TP: 1023 \\
\hline
\end{tabular}

The combination of the above Deep Learning implementations leads to the development of the Data Fusion based Neural Network, which is shown in Figure 3. Training curves are presented in Figure 8. Training process ends in the 19th Epoch due to the Early Stopping condition, because there is no improvement of the loss function, while in the 16th the minimum value of BCE is observed. The F1 metric performance results in test Dataset are $98.7 \%$, while Table 5 presents the confusion matrix on test dataset. Therefore, the DNN 
can be used to classify the potential regions as suitable or not for WEC installation. The final decision depends upon the combination of the presence or absence of algae and wave energy assessment. According to Figure 9, the Binary Classifier works efficiently because it identifies the potential regions of the test dataset with high accuracy. In particular, it correctly classifies both suitable sites, and non-suitable ones that they are points with algae presence or with low wave energy. Both the prediction of DNN and the actual labels are shown in order to understand the final decision.

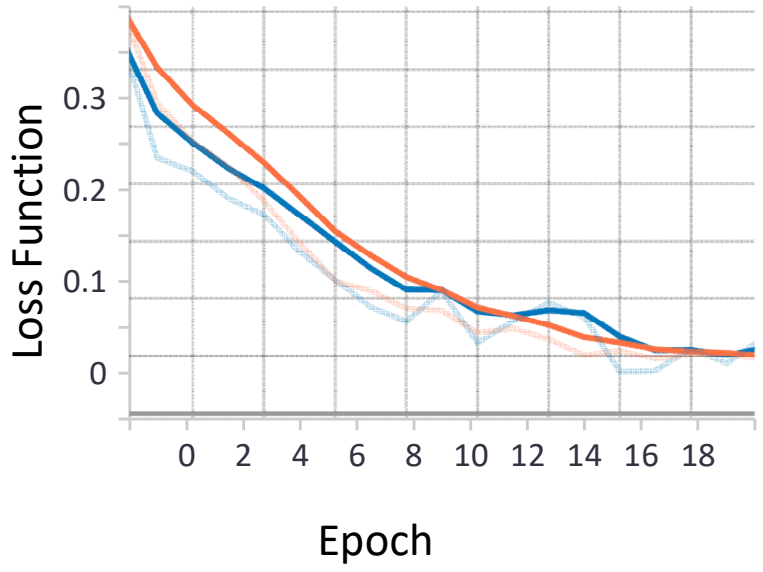

(a)

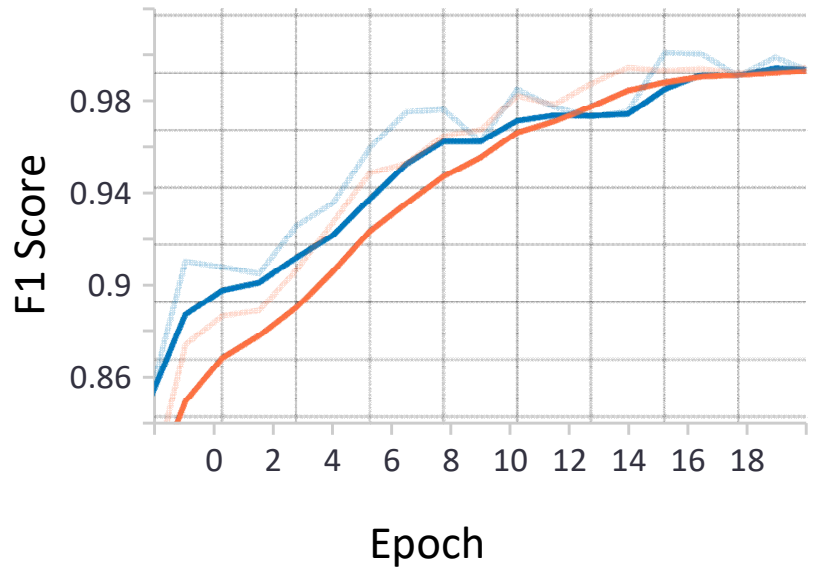

(b)

Figure 8. Data Fusion based Neural Network performance during training process. (a) Binary Cross Entropy Loss for both training (orange) and validation (blue) datasets; (b) F1 score for both training and validation datasets.

Table 5. Confusion Matrix of Neural Network that classifies the suitability of potential WEC installation regions.

\begin{tabular}{ccc}
\hline & Predicted-Class 0 & Predicted-Class 1 \\
\hline Actual-Class 0 & TN: 799 & FP: 10 \\
Actual-Class 1 & FN: 7 & TP: 847 \\
\hline
\end{tabular}
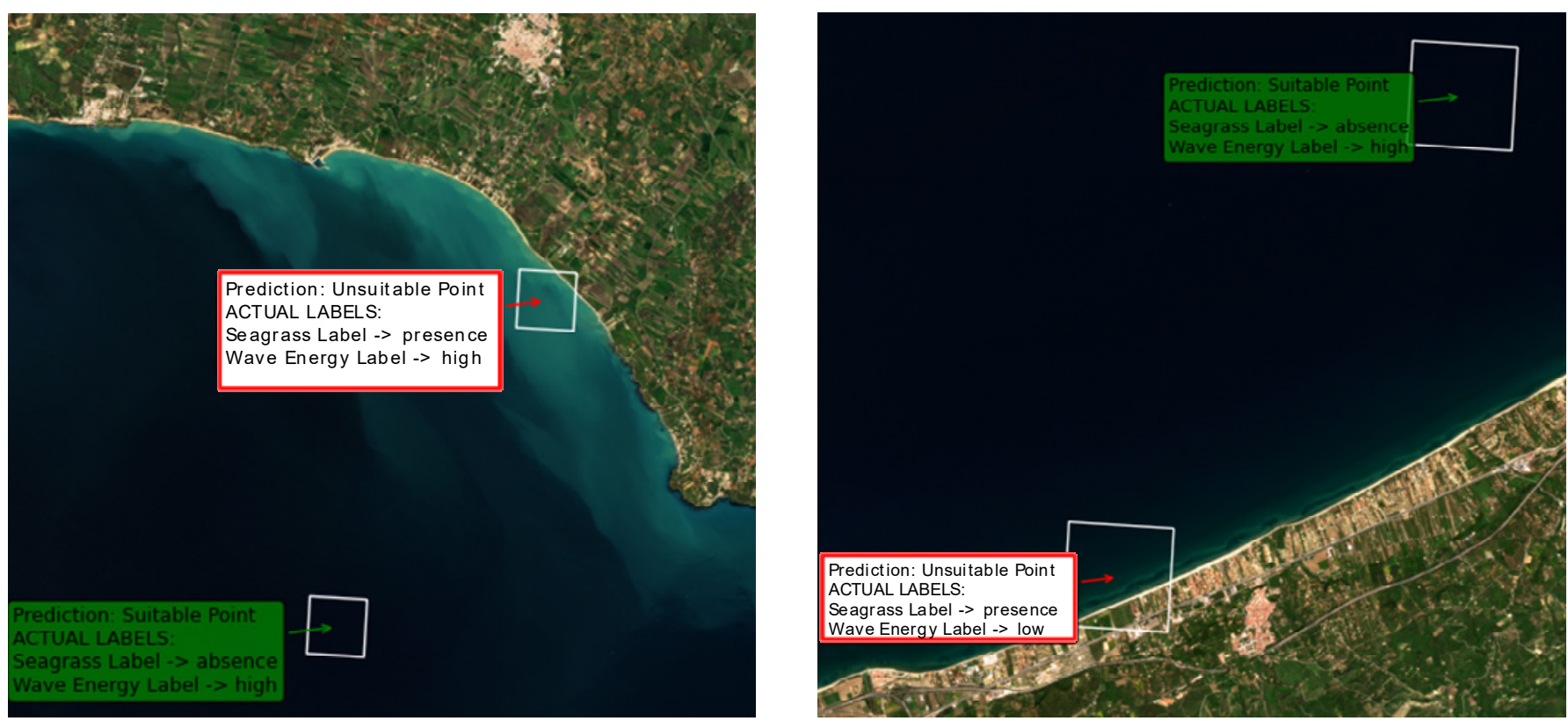

Figure 9. Data Fusion based Neural Network predictions on test dataset. 


\subsection{Multitask Data Fusion Based Neural Network}

Proceeding to the development of Multitask Data Fusion based Neural Network (Figure 5), we integrate the land cover classification task to our model. The architecture consists of two outputs. The first output is for the binary prediction of the potential region as suitable or not suitable for the WEC system installation and the second for the prediction of One-Hot vector of limitations, in this case land use classes, algae detection and the wave energy class. Regarding Figure 10, in the 24th Epoch the training ends, while in the 15th the lower value of the BCE is observed. We defined as the loss function and metric for both outputs BCE-F1 Score. The overall F1 Score is $94 \%$.

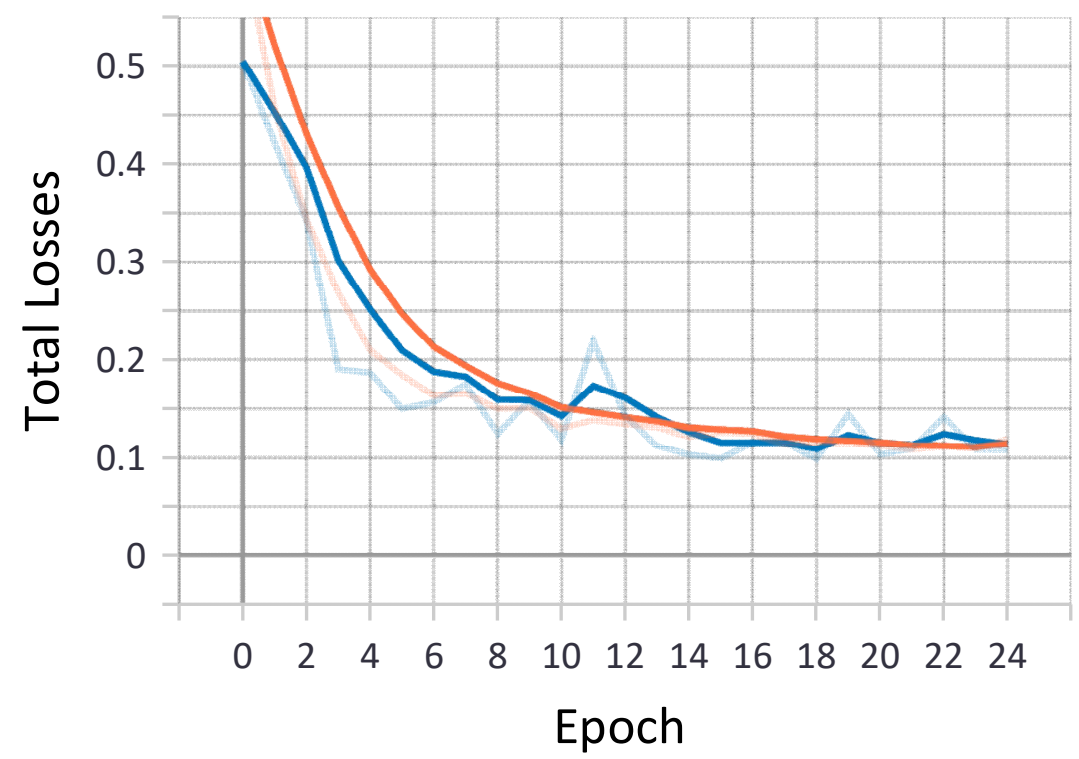

Figure 10. Total training and validation losses for Multitask Data Fusion based Neural Network.

For potential WEC installation points classified as True Positive or True Negative, the corresponding One-Hot Label is predicted correctly as well. Figure 11 shows the results on test data. As observed, for each region, both the Multilabel vector containing the detected classes is predicted, as well as the designation as suitable or not. As the two loss functions are optimized at the same time, there is an interdependence between the outputs, which is the objective of our approach. The difference between the F1 score of the two Neural Networks arises because in Mode II we have larger prediction complexity due to the dependence of the two outputs and the recognition of land use classes.

\subsection{Expreriments of Methodology Application Case Study}

We applied the proposed methodology to the geographical area of Figure 12a. By defining the geographical coordinates of the selected bounding box on the map, data extraction and preprocessing is performed, as shown in Section 2.1. In particular, we receive the Sentinel-2 images and we define the Grid of the potential WEC installation points. For each point, the Patch of the image is extracted and the wave height and period time series are obtained from the Era- 5 dataset. In Figure 12b, the gaps observed, are regions that were automatically excluded because they are out of the desired water depth range. 

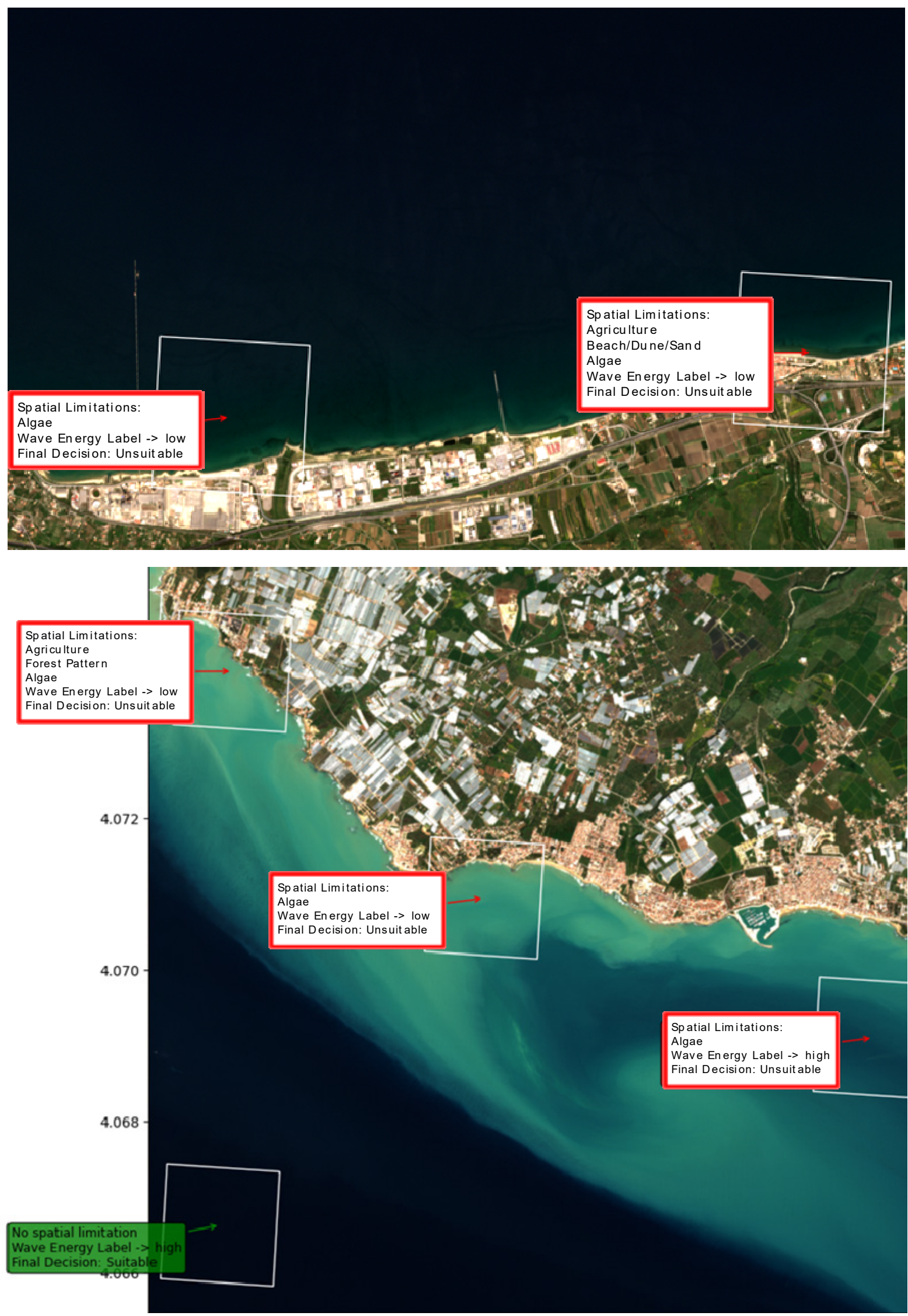

Figure 11. Multitask Data Fusion based Neural Network predictions on test dataset. 


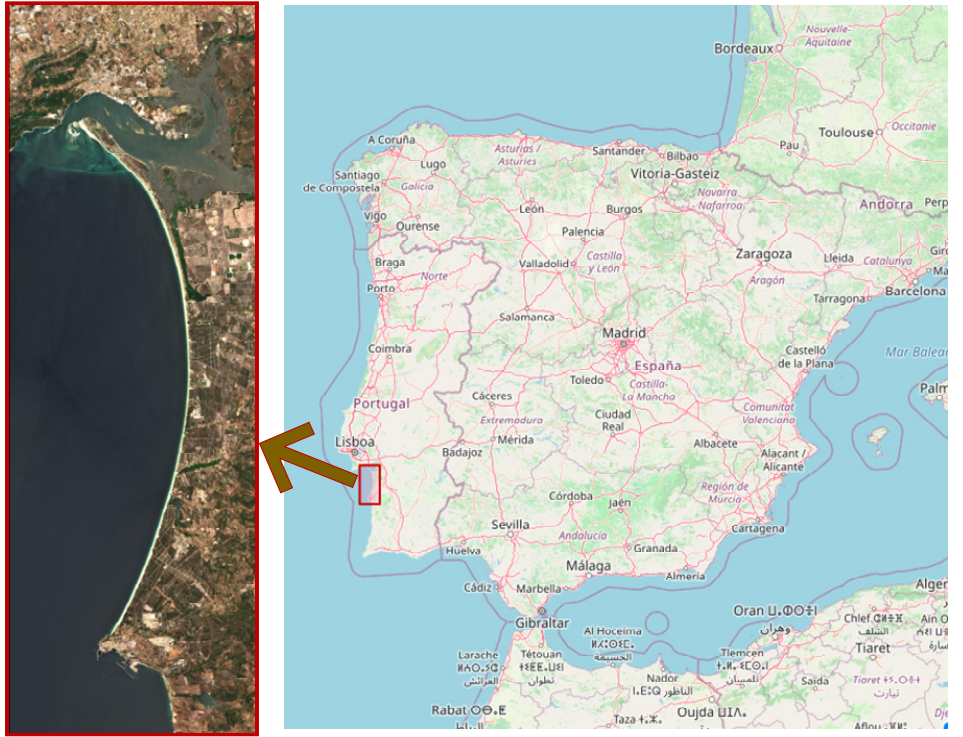

(a)

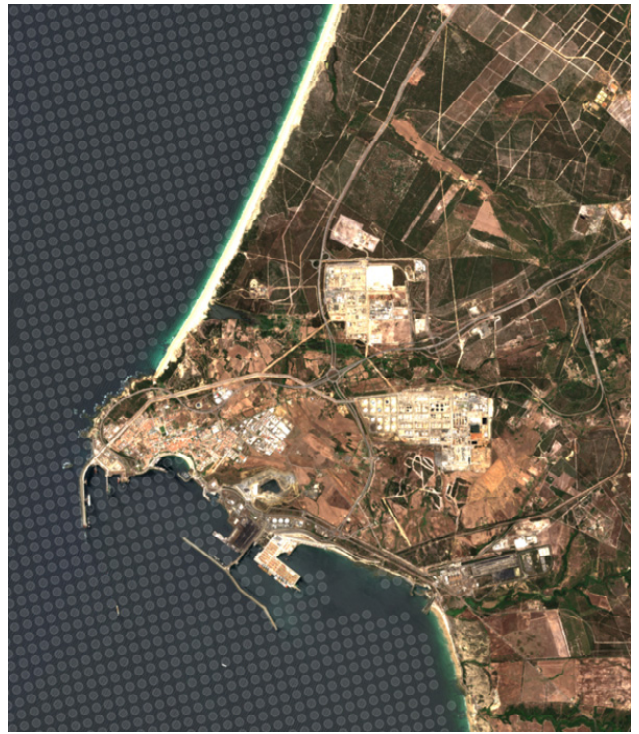

(b)

Figure 12. (a) Case study; Sines, Portugal; (b) potential WEC installation points of interest.

In Mode I, the suitability map is first derived based on the algae detection and wave energy assessment. Neural Network predictions are presented in Figures 13a and 14a. Then, the available land use/land cover data are fetched from the CLC dataset and depending on the geographical constraints that arise, the final decision is formed, as Figures $13 \mathrm{~b}$ and 14b show. The areas highlighted in red color are excluded, while in green the suitable for WEC system sites are shown.

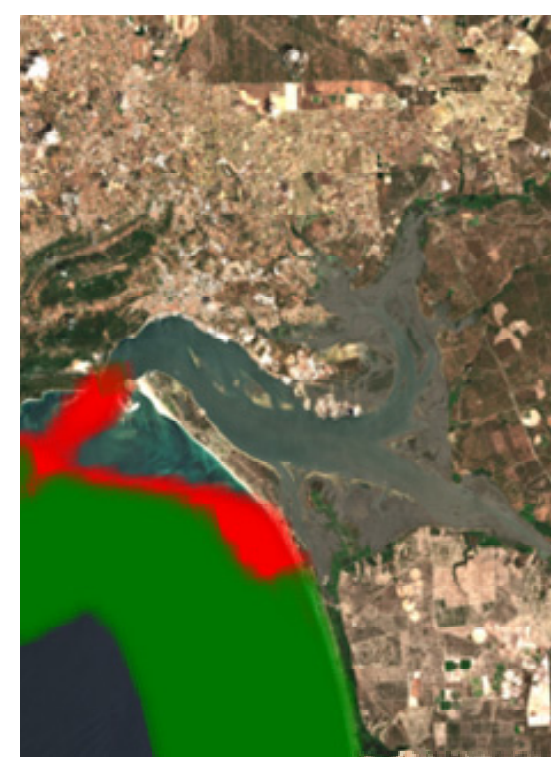

(a)

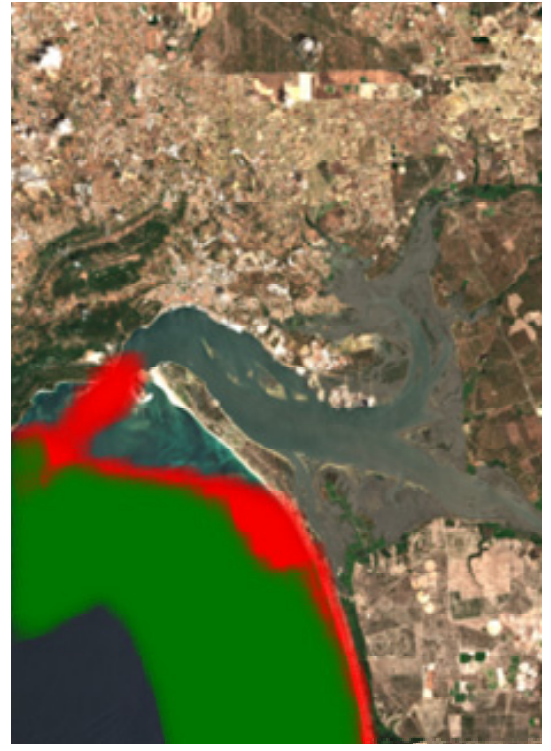

(b)

Figure 13. (a) Neural Network Prediction; (b) final decision considering land use data. 


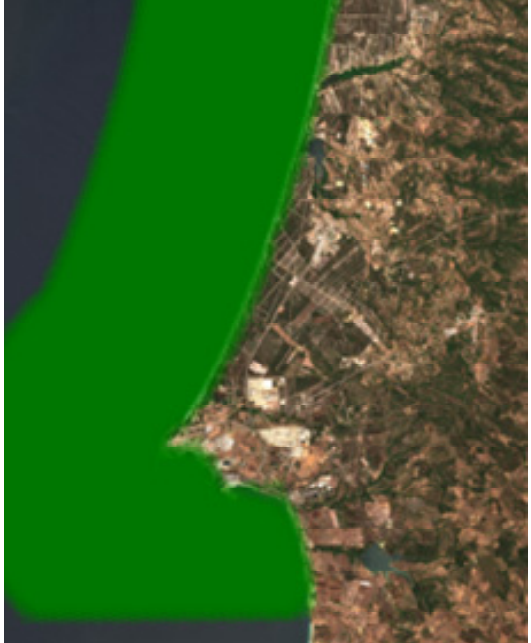

(a)

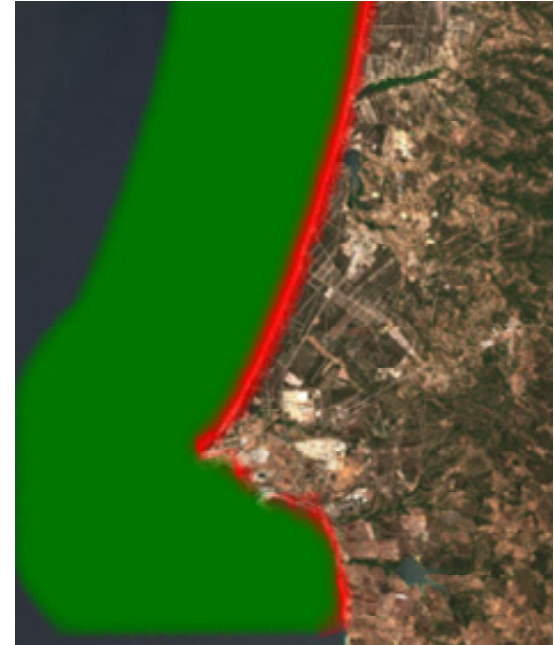

(b)

Figure 14. (a) Neural Network Prediction; (b) final decision considering land use data.

Utilizing the Multitask Data Fusion based Neural Network in Mode II, the suitability map is generated directly from the DNN predictions and specially the output of Binary Classification task (Figures 15a and 16a). In addition, One-Hot label, which results from the second output of the Neural Network, appears for each of the potential regions. In this way, the cause for which the area was excluded from the study becomes known. In Figures $15 \mathrm{~b}$ and $16 \mathrm{~b}$ there is a sample of predictions of the second task.

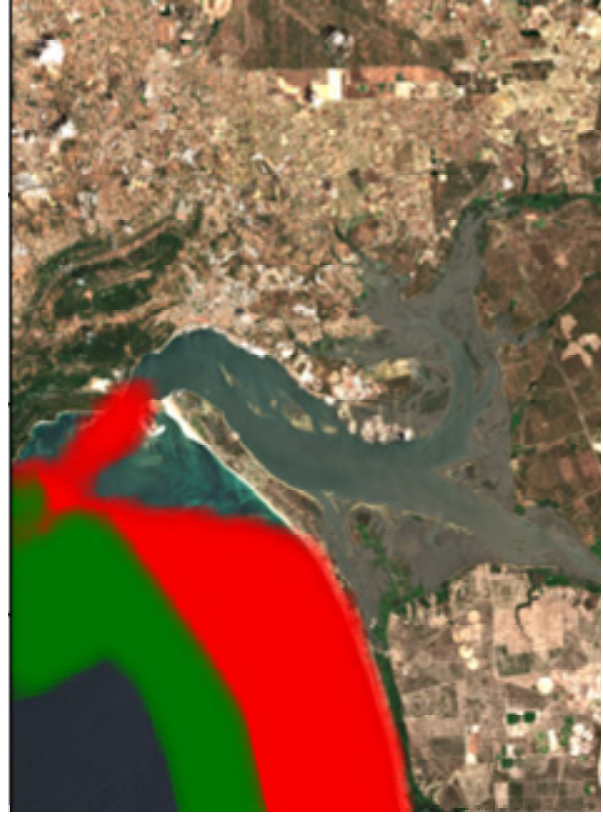

(a)

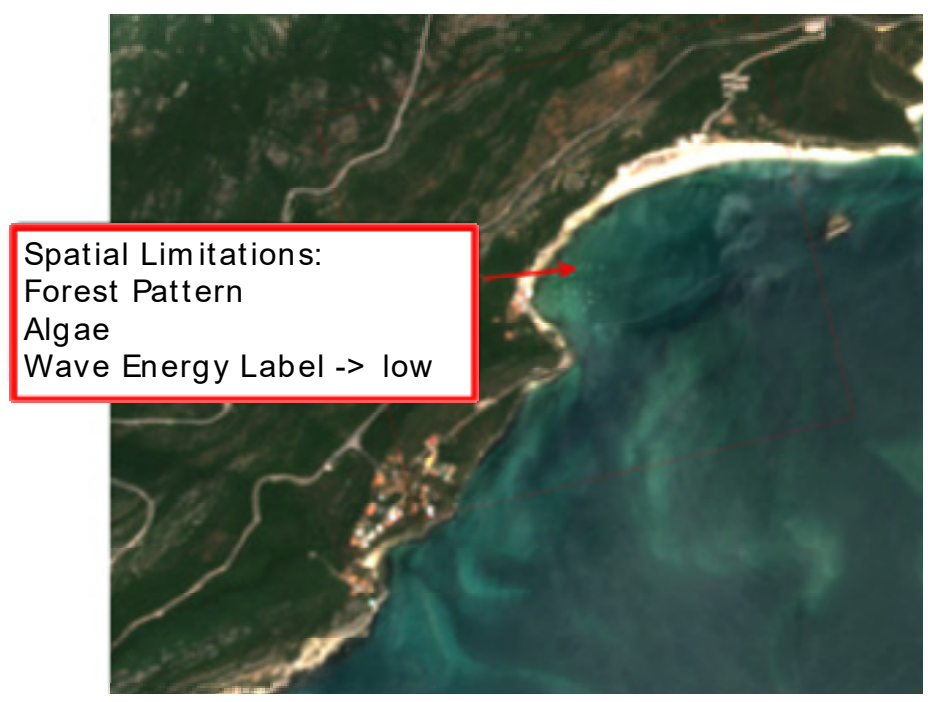

(b)

Figure 15. (a) Suitability map-Neural Network Predictions; (b) zoom to observe Multilabel Classification results. 


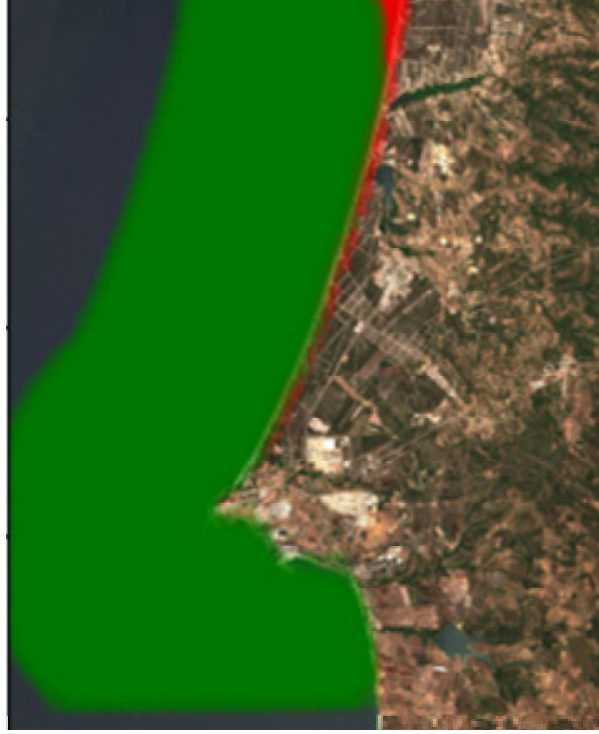

(a)

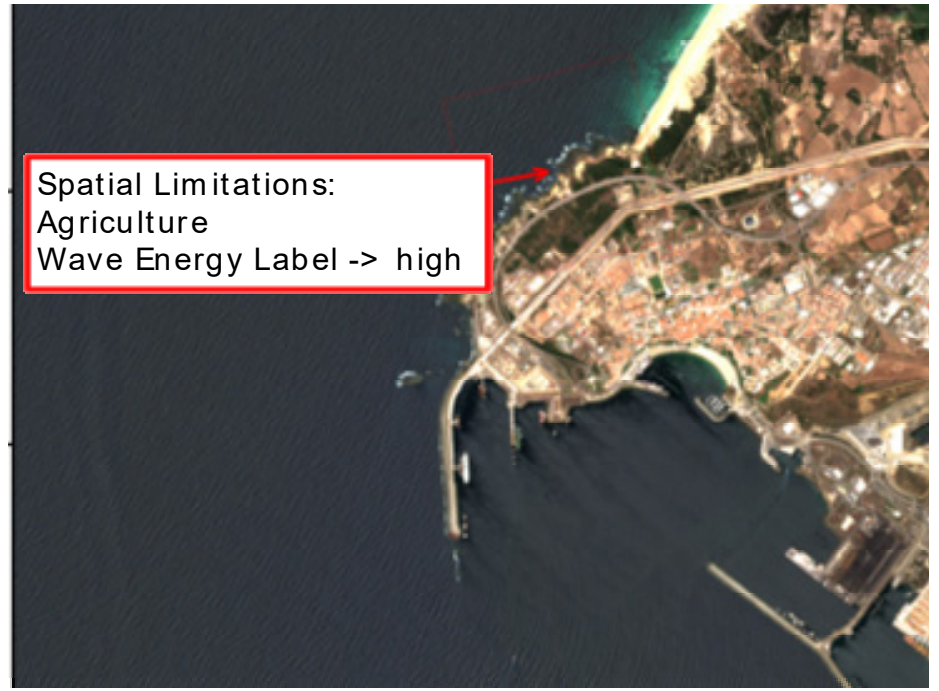

(b)

Figure 16. (a) Suitability map-Neural Network Predictions; (b) zoom to observe Multilabel Classification results.

Both Modes make common predictions of offshore points of interest with algae presence. In Figures 13a and 15a our models classify differently the wave energy potential in some offshore points of interest due to FN predictions of Neural Network in Mode II, because the Binary Classification task depends on the Multilabel Classification task. In the majority of nearshore regions, which are affected by land use, there is an agreement between the two approaches. Nevertheless, we notice in Figures 14a and 16a that the Multitask DNN does not properly classify coastal areas in the southern part, because in order to identify a land use class in the satellite image, the latter must correspond to a significant percentage of the coast and be clearly visible. When using Mode I, land use classes are found using the spatial intersection operation, which always implies their correct detection. Nobre et al. [44] focus in this geographical area during their study. Comparison of the results with the proposed approach can only be done for offshore points, because the authors reject the areas near the coast. Although the estimate of the potential wave energy is made in a different way and in the present paper we detect seagrass in marine areas, there is an agreement of the results to a very large extent.

\section{Conclusions and Discussion}

This paper presents a new, Deep Learning-based methodology to automate the process of WEC optimal positioning. The presented models work with freely available satellite images, as well as Climate Reanalysis data. The methodology is applied through two different approaches. In Mode I, land use data are received from CLC dataset, while in the second method they are extracted from the satellite images in order to avoid additional geographic limitations. At the end of the training process, the model being developed is capable of identifying the spatial restrictions in satellite images, including algae patterns that are dynamically changing features. At the same time, the system estimates the wave energy potential by treating it as a dynamic phenomenon, which is characterized by non-predictable temporal variability. In addition, our model can identify the differences in temporal variability of multiple locations. In this way, it is confirmed that merging heterogeneous data works efficiently in solving complex problems. Thus, it turns out that CNNs are efficient in both image and time series recognition.

In this paper, we initiate a new method for the spatial positioning of WEC that is based in recognition of geospatial constraints which are dynamically changing patterns in marine areas. Thus, the main limitation of this study is the fact that additional dynamic classes can be added to satellite image recognition task. Under this rationale, the proposed 
model can be generalized to include several image-related dynamic phenomena. In marine areas there are characteristics that change in time, so their identification is difficult without Machine Learning or costly monitoring. In addition, the main technical challenge of this study is the fact that the examination of dynamic geospatial and technical restrictions should be combined in terms of Levelized Cost of Energy (LCOE) of wave energy and GIS methods [45]. Regarding land use, the interconnection of the proposed system with an API such as Google Maps/Places or Open Street Map could be efficient because these platforms contain data that are frequently updated. Finally, in addition to assessing climatic conditions, a third branch can be added to the proposed Neural Network for time series forecasting.

Author Contributions: Conceptualization, G.B., G.S. and P.P.; methodology, G.B., G.S. and P.P.; software, G.B.; validation, G.B. and G.S.; formal analysis, G.B.; resources, G.B. and P.P.; data curation, G.B.; writing—original draft preparation, G.B.; writing—review and editing, G.B., G.S. and P.P.; supervision, G.S. and P.P.; All authors have read and agreed to the published version of the manuscript.

Funding: This research received no external funding.

Institutional Review Board Statement: Not applicable.

Informed Consent Statement: Not applicable.

Data Availability Statement: UN Environment Programme World Conservation Monitoring Centre provides open-access data about the global distribution of seagrass. Copernicus Land Monitoring Service produced the Corine Land Cover Dataset within the frame of CORINE Land Cover Programme, which is coordinated by the European Environment Agency (EEA) (https://land. copernicus.eu/pan-european/corine-land-cover, accessed on 10 September 2021). Furthermore, Copernicus Proramme and Open Acess Hub provide publicly available Sentinel 2 satellite imagery (https:/ / scihub.copernicus.eu/, accessed on 10 September 2021). Concerning time series data, the ERA5 is the ECMWF reanalysis dataset for the global climate and weather. These data are available through the Climate Data Store (https:/ / cds.climate.copernicus.eu/cdsapp\#!/dataset/reanalysisera5-single-levels?tab=overview, accessed on 10 September 2021).

Conflicts of Interest: The authors declare no conflict of interest.

\section{Index of Abbreviations}

$\begin{array}{ll}\text { WEC } & \text { Wave Energy Converters } \\ \text { MSP } & \text { Marine Spatial Planning } \\ \text { EEZ } & \text { Exclusive Economic Zone } \\ \text { SWAN } & \text { Simulating Waves Nearshore } \\ \text { GIS } & \text { Geographic Information Systems } \\ \text { MCDM } & \text { Multiple-criteria decision-making } \\ \text { FI } & \text { Feasibility Index } \\ \text { ANN } & \text { Artificial Neural Network } \\ \text { ML } & \text { Machine Learning } \\ \text { DNN } & \text { Deep Neural Networks } \\ \text { CNN } & \text { Convolutional Neural Network } \\ \text { CDS } & \text { Climate Data Store } \\ \text { CLC } & \text { Corine Land Cover } \\ \text { CV } & \text { Coefficient of Variation } \\ \text { GAP } & \text { Global Average Pooling } \\ \text { BCE } & \text { Binary Cross Entropy } \\ \text { TP } & \text { True Positive } \\ \text { TN } & \text { True Negative } \\ \text { FP } & \text { False Positive } \\ \text { FN } & \text { False Negative } \\ \text { LCOE } & \text { Levelized Cost of Energy } \\ & \end{array}$




\section{References}

1. Panwar, N.; Kaushik, S.; Kothari, S. Role of renewable energy sources in environmental protection: A review. Renew. Sustain. Energy Rev. 2011, 15, 1513-1524. [CrossRef]

2. Bilgen, S. Global warming and renewable energy sources for sustainable development: A case study in Turkey. Renew. Sustain. Energy Rev. 2008, 12, 372-396. [CrossRef]

3. Commision, E. The 2030 Climate and Energy Framework; Palgrave Macmillan: Cham, Switzerland, 2013.

4. The European Ocean Energy Association (EU-OEA). Oceans of Energy-European Ocean Energy Roadmap 2010-2050; EU-OEA: Brussels, Belgium, 2010.

5. Pérez-Collazo, C.; Greaves, D.; Iglesias, G. A review of combined wave and offshore wind energy. Renew. Sustain. Energy Rev. 2015, 42, 141-153. [CrossRef]

6. Commision, E. Maritime Spatial Planning in the EU-Achievements and future development. Communication from the Commision to the European Parliament, the Council, the European Economic and Social Commitee and the Committe of the Regions; European Commission: Brussels, Belgium, 2010.

7. Farkas, A.; Degiuli, N.; Martić, I. Assessment of Offshore Wave Energy Potential in the Croatian Part of the Adriatic Sea and Comparison with Wind Energy Potential. Energies 2019, 12, 2357. [CrossRef]

8. Nilsson, E. Characterization of Wave Energy Potential for the Baltic Sea with Focus on the Swedish Exclusive Economic Zone. Energies 2019, 12, 793. [CrossRef]

9. Veigas, M.; Lopez, M.; Iglesias, G. Assessing the optimal location for a shoreline wave energy converter. Appl. Energy 2014, 132, 404-411. [CrossRef]

10. Vicinanza, D. The SSG Wave Energy Converter: Performance, Status and Recent Developments. Energies 2012, 5, 193-226. [CrossRef]

11. Amarouche, K. Wave energy resource assessment along the Algerian coast based on 39-year wave hindcast. Renew. Energy 2020, 153, 840-860. [CrossRef]

12. Fairley, I. A classification system for global wave energy resources based on multivariate clustering. Appl. Energy 2020, $262,114515$. [CrossRef]

13. Aydin, N.Y.; Kentel, E.; Duzgun, H.S. GIS-based site selection methodology for hybrid renewable energy systems: A case study from western Turkey. Energy Convers. Manag. 2013, 70, 90-106. [CrossRef]

14. Castro-Santos, L. Planning of the installation of offshore renewable energies: A GIS approach of the Portuguese roadmap. Renew. Energy 2019, 132, 1251-1262. [CrossRef]

15. Galparsoro Iza, I. A Marine Spatial Planning Approach to Select Suitable Areas for Installing Wave Energy Converters (WECs), on the Basque Continental Shelf (Bay of Biscay). Coast. Manag. 2012, 40, 1-19. [CrossRef]

16. Vasileiou, M.; Loukogeorgaki, E.; Vagiona, D.G. GIS-based multi-criteria decision analysis for site selection of hybrid offshore wind and wave energy systems in Greece. Renew. Sustain. Energy Rev. 2017, 73, 745-757. [CrossRef]

17. Ghosh, S. Wave Energy Potential Site Selection Based on MCDM and Neural Network Analysis. In Application of Geographical Information Systems and Soft Computation Techniques in Water and Water Based Renewable Energy Problems; Springer: Singapore, 2018; pp. 107-120.

18. Effrosynidis, D.; Arampatzis, A.; Sylaios, G. Seagrass detection in the mediterranean: A supervised learning approach. Ecol. Inform. 2018, 48, 158-170. [CrossRef]

19. Traganos, D. Towards Global-Scale Seagrass Mapping and Monitoring Using Sentinel-2 on Google Earth Engine: The Case Study of the Aegean and Ionian Seas. Remote Sens. 2018, 10, 1227. [CrossRef]

20. Li, X. Deep-learning-based information mining from ocean remote-sensing imagery. Natl. Sci. Rev. 2020, 7, 1584-1605. [CrossRef]

21. Kalogerakis, C.; Koutroulis, E.; Lagoudakis, M.G. Global MPPT Based on Machine-Learning for PV Arrays Operating under Partial Shading Conditions. Appl. Sci. 2020, 10, 700. [CrossRef]

22. Jayalakshmi, N. Novel Multi-Time Scale Deep Learning Algorithm for Solar Irradiance Forecasting. Energies 2021, 14, 2404. [CrossRef]

23. Jin, X.-B. Deep-Learning Forecasting Method for Electric Power Load via Attention-Based Encoder-Decoder with Bayesian Optimization. Energies 2021, 14, 1596. [CrossRef]

24. Peng, X. Short-Term Wind Power Prediction for Wind Farm Clusters Based on SFFS Feature Selection and BLSTM Deep Learning. Energies 2021, 14, 1894. [CrossRef]

25. Castello, R. Deep learning in the built environment: Automatic detection of rooftop solar panels using Convolutional Neural Networks. J. Physics Conf. Ser. 2019, 1343, 012034. [CrossRef]

26. Lee, S. Deep Roof: A Data-driven Approach For Solar Potential Estimation Using Rooftop Imagery. In Proceedings of the 25th ACM SIGKDD International Conference on Knowledge Discovery \& Data Mining, Anchorage, AK, USA, 8 August 2019; pp. 2105-2113.

27. Cao, R. Deep learning-based remote and social sensing data fusion for urban region function recognition. ISPRS J. Photogramm. Remote Sens. 2020, 163, 82-97. [CrossRef]

28. ESA. Copernicus Sentinel Data 2018-2021; ESA: Paris, France; pp. 2018-2021.

29. British Oceanographic Data Centre. GEBCO Digital Atlas: Digital version of the IOC/IHO General Bathymetric Chart of the Oceans (GEBCO); British Oceanographic Data Centre: Liverpool, UK, 1994. 
30. Hersbach, H.; Bell, B.; Berrisford, P.; Biavati, G.; Horányi, A.; Muñoz Sabater, J.; Nicolas, J.; Peubey, C.; Radu, R.; Rozum, I.; et al. ERA5 hourly data on single levels from 1979 to present. Copernic. Clim. Chang. Serv. Clim. Data Store 2018.

31. EEA. Corine Land Cover (CLC); EEA: Copenhagener, Denmark, 2018.

32. Short, F.; Green, E. World Atlas of Seagrasses; University of California Press: Berkeley, CA, USA, 2003; ISBN 0-520-24047-2.

33. UNEP-WCMC; Short, F. Global Distribution of Seagrasses (Version 7.1). Seventh Update to the Data Layer Used in Green and Short (2003); UN Environment World Conservation Monitoring Centre; Cambridge, UK, 2021.

34. DiCarlo, J.J.; Zoccolan, D.; Rust, N.C. How Does the Brain Solve Visual Object Recognition? Neuron 2012, 73, 415-434. [CrossRef] [PubMed]

35. Gonzalez, R.C.; Woods, R.E. Digital Image Processing; Pearson: London, UK, 2018; ISBN 0133356728/9780133356724.

36. LeCun, Y. Backpropagation Applied to Handwritten Zip Code Recognition. Neural Comput. 1989, 1, 541-551. [CrossRef]

37. Yamashita, R. Convolutional neural networks: An overview and application in radiology. Insights Imaging 2018, 9, 611-629. [CrossRef] [PubMed]

38. Fawaz, H.I.; Forestier, G. Deep learning for time series classification: A review. Data Min. Knowl. Discov. 2019, 33, 917-963. [CrossRef]

39. Zhao, B. Convolutional neural networks for time series classification. J. Syst. Eng. Electron. 2017, 28, 162-169. [CrossRef]

40. Kingma, D.P.; Ba, J. Adam: A method for stochastic optimization. In Proceedings of the International Conference Learn. Represent. (ICLR), San Diego, CA, USA, 5-8 May 2015.

41. Sokolova, M.; Lapalme, G. A systematic analysis of performance measures for classification tasks. Inf. Process. Manag. 2009, 45, 427-437. [CrossRef]

42. Goutte, C.; Gaussier, E. A Probabilistic Interpretation of Precision, Recall and F-Score, with Implication for Evaluation. In Proceedings of the 27th European Conference on IR Research, ECIR 2005, Santiago de Compostela, Spain, 21-23 March 2005; Volume 3408, pp. 345-359.

43. Arellano-Verdejo, J.; Hernandez, H.E.L.; Cabanillas-Terán, N. ERISNet: Deep neural network for Sargassum detection along the coastline of the Mexican Caribbean. PeerJ 2019, 7, e6842. [CrossRef]

44. Nobre, A. Geo-spatial multi-criteria analysis for wave energy conversion system deployment. Renew. Energy 2009, 34, 97-111. [CrossRef]

45. Castro-Santos, L. The Levelized Cost of Energy (LCOE) of wave energy using GIS based analysis: The case study of Portugal. Int. J. Electr. Power Energy Syst. 2015, 65, 21-25. [CrossRef] 